http://jmscr.igmpublication.org/home/ ISSN (e)-2347-176x ISSN (p) 2455-0450 crossref DOI: https://dx.doi.org/10.18535/jmscr/v8i10.48

\title{
A study of Correlation between the Level of Suicidal Intent and Mode of Attempt
}

\author{
Authors \\ Dr Jayaraman $\mathbf{S}^{1}$, Dr Asokkumar $\mathbf{M}^{2}$, Dr Gandhibabu $\mathbf{R}^{3}$ \\ ${ }^{1}$ Postgraduate, Department of Psychiatry, Rajah Muthiah Medical College and Hospital, Annamalai \\ University, Chidambaram, Tamilnadu \\ ${ }^{2}$ Professor and Head, Department of Psychiatry, Rajah Muthiah Medical College and Hospital, Annamalai \\ University, Chidambaram, Tamilnadu \\ ${ }^{3}$ Professor, Department of Psychiatry, Rajah Muthiah Medical College and Hospital, Annamalai University, \\ Chidambaram, Tamilnadu
}

\begin{abstract}
Suicide is becoming the leading cause of death in most countries in certain population groups. It is the high time to know the background picture of it. This study was conducted to know in detail about the various modes of suicide attempt in persons admitted in Hospital, and consecutive 90 subjects were assessed by self-innovated proforma. It also assess the level of suicide attempt in these subjects with the becks suicide intent scale. These details can be compared with previous studies to gain knowledge of it and use those in preventive strategies.
\end{abstract}

Results: Out of 90 subjects 69 (76.7\%) attempted by poisoning, 11 (12.2\%) by hanging, $8(8.9 \%)$ by tablet overdose and $2(2.2 \%)$ self-inflicted. In poisoning, rat killer and organophosphorus poison by 18 (26.1\%) each. Followed by ant killer poison by 10 (14.5\%), kerosene by 8(11.6\%), oleander seed poison by 6 $(8.7 \%)$, abrus seed by $4(5.8 \%)$ and $5(7.2 \%)$ by other mode of poisoning. In 90 subjects 42 (46.7\%) has low intent, 36 (40.0) has medium and 12 (13.3\%) had low intent.

Conclusion: The intent is low in suicide attempt compared to death by suicide, where the intent would be high. Poisoning is the leading mode of attempt with domestically available poisons (rat killer/ant killer/kerosene). The accessibility to these products can be limited to reduce the suicidal behaviors.

Keywords: Suicide attempt, intent, mode of attempt.

\section{Introduction}

In India, in 2018, 134,516 people die by suicide. It is estimated that 1 in 60 persons are affected by suicide $^{1}$. For every death by suicide, on an average, 25 people attempt to die by suicide. In 2016, approximately 5.75 million people attempted to die by suicide in India ${ }^{13,14}$.

There are various methods employed to attempt suicide, which depends on various factors such as availability, accessibility and socioeconomic factors. Common methods used in developed countries differ from developing countries.

According to NCRB data of Accidental Deaths and Suicide in India 2018 report, suicide by hanging $51.5 \%$, poison $26.7 \%$, drowning $4.9 \%$, self-immolation $4.4 \%$ were prominent means of committing suicides during $2018^{1}$. 
In 2012, Rajiv Radhakrishnan et al, stated that "pesticide is the most common method as it is available in India $^{2,3}$. Men more likely use organophosphorus poison and women more likely use plant poisons ${ }^{4}$. Other methods were hanging, drug overdose, drowning, jumping from height".

Poisoning was frequently used by attempters without any psychiatric disorders, attempting for the first time or adolescent age group people. Substance abusers and psychiatric patients more commonly use physical methods ${ }^{5}$.

Self-immolation is used as method of suicide in India more commonly than other countries. Because, it has its roots in to Indian history. Sati and Jauhar in ancient India is seen as an escape from hardships and humiliation ${ }^{2}$. But this reason applies to most of the suicidal attempt.

In Sati, a widow sacrifices herself by sitting atop her deceased husband's funeral pyre ${ }^{6}$. Jahuar is the act of mass self-immolation by women to avoid captive enslavement and rape by foreign invaders, when facing certain defeat during a war? ${ }^{7}$.

Bastia BK et al states that "Most of the female suicidal hanging happened at home, in the daytime in contrast to men. The materials used for hanging precluded any scope of restriction. Some the stresses were reported specific to genders; e.g., dowry, illicit relationship of spouse, illegitimate pregnancy, not having children and no settlement of marriage were specific to women; while unemployment was solely reported in male suicides".

Married females, unmarried males and students were predominantly seen in the study that used the psychological autopsy method to find the factors associated with suicidal hanging. Based on this, the vulnerability can be identified early and close monitoring at home and specific support can be provided to prevent hanging ${ }^{8}$.

\section{Intent}

According to the model suggested O'Connell et al., "suicide is a serial process encompassing ideation, planning, attempts, and completion"10.
Attempted suicide is regarded as the important predictor of future suicide attempts or death by it because attempted suicide is the phase prior to death by suicide ${ }^{11}$.

People with high intent of suicide have the characteristics that is similar to the people died due to completing suicide. By identifying these characteristics, important baseline data can be obtained that is required to develop appropriate interventions in preventing suicide ${ }^{12}$.

According to Alvi T et al., in January 2017, 43\% of suicide attempters had high level of intent, $33 \%$ had medium intent and 24\% had low level of suicidal intent ${ }^{9}$. The intend of this study is to find the various mode of suicide attempts and the intent of the attempters admitted in the hospital.

\section{Materials and Methods \\ Study Setting}

Study will be conducted in Rajah Muthiah Medical College Hospital (RMMCH), Annamalai University, Annamalai Nagar, Chidambaram, Tamil Nadu.

\section{Sample}

Consecutive suicide attempters who are all admitted in RMMC, from 01.01.2019 and referred to Department of Psychiatry for assessment.

\section{Study Design}

Observational study - Cross sectional study

\section{Inclusion Criteria}

- Suicide attempters of both sexes are included

- Suicide attempters who fulfilled the criteria

- Suicide attempters who are willing to participate in the study

\section{Exclusion Criteria}

- Suicide attempters who are not willing to participate in study

- Suicide attempters those who are seriously ill and could not participate in the study

\section{Interview}

Study will be conducted on the 90 suicide attempters those who are admitted in hospital and 
referred for psychiatric evaluation. Will be evaluated for psychosocial, situational and clinical risk factors using following materials

\section{Materials Used}

1. Self-innovated proforma to elicit the Socio Demographic data

2. Becks suicide intent scale

3. ICD-10 - classification of Mental and Behavioral disorders

\section{Results}

Table 1: Distribution according to the mode of attempt among the cases

\begin{tabular}{|l|c|c|}
\hline \multirow{2}{*}{ Mode of attempt } & \multicolumn{2}{|c|}{ Cases } \\
\cline { 2 - 3 } & $\mathbf{N}$ & $\mathbf{\%}$ \\
\hline Poisoning & 69 & 76.7 \\
\hline Hanging & 11 & 12.2 \\
\hline Tablet over dose & 8 & 8.9 \\
\hline Self-inflicted & 2 & 2.2 \\
\hline
\end{tabular}

In table 1, out of 90 subjects assessed $69(76.7 \%)$ attempted suicide by poisoning. 11 (12.2\%) attempted suicide by hanging, $8(8.9 \%)$ by tablet overdose and $2(2.2 \%)$ self-inflicted.

Table 2: Distribution according to the type of poisoning among those who attempted suicide by poisoning

\begin{tabular}{|l|c|c|}
\hline $\begin{array}{l}\text { Type of } \\
\text { poisoning }\end{array}$ & $\begin{array}{c}\text { Frequency } \\
(\mathbf{n = 6 9 )}\end{array}$ & Percentage \\
\hline Rat killer & 18 & 26.1 \\
\hline Abrus seed & 4 & 5.7 \\
\hline OPC & 18 & 26.1 \\
\hline Kerosene & 8 & 8.9 \\
\hline Ant killer & 10 & 11.6 \\
\hline Oleander seed & 6 & 8.7 \\
\hline Others & 5 & 7.2 \\
\hline
\end{tabular}

Among the subjects who had attempted suicide by poisoning, rat killer poison and organophophorus poisoning tops the list with 18 (26.1\%) each. Followed by ant killer poison by 10 (14.5\%), kerosene by $8(11.6 \%)$, oleander seed poison by 6 (8.7\%), abrus seed by $4(5.8 \%)$ and $5(7.2 \%)$ by other mode of poisoning
Fig 8: Pie chart showing the distribution of intent among the study participants who had attempted suicide.

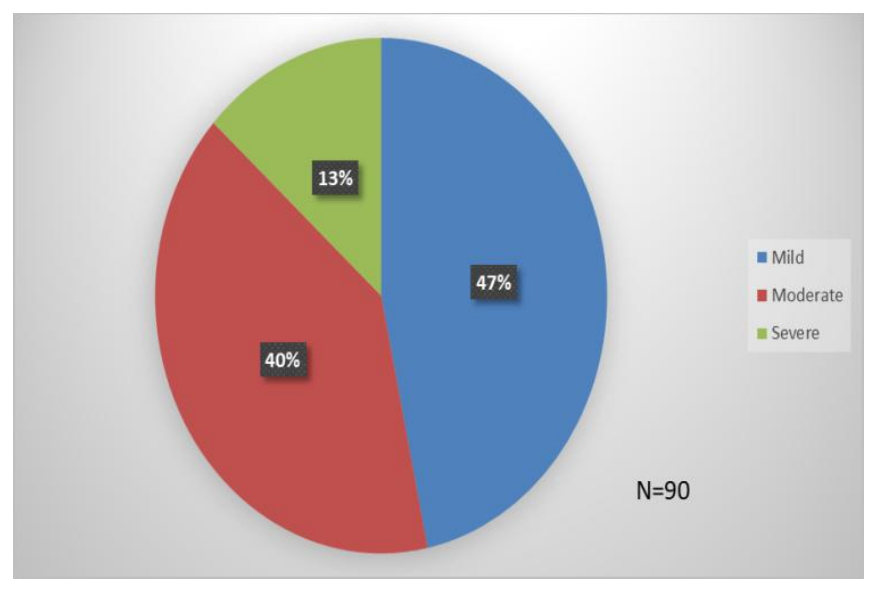

The subjects $(\mathrm{n}=90)$ were assessed with becks suicide intent scale to know the level of intent of suicide attempt. In that, $42(46.7 \%)$ persons fall in the category of mild (low intent) with sore less than 19. In the category of moderate (medium intent) with sore of $20-28,36(40.0 \%)$ were there. With score of more than 29, 12 (13.3\%) persons comes under severe (high intent).

\section{Discussion}

In this study the 90 subjects admitted in hospital for suicide attempt were questioned regarding the mode of attempt and found that $76.7 \%$ of the attempt were by poisoning. Remaining were by hanging $12.2 \%$, tablet overdose in $8.9 \%$, and selfinflicted by $2.2 \%$ of the subjects. This result does not matches with the NCRB 2018 data $^{1}$, which shows hanging with $51.5 \%$ and poisoning by $26.7 \%$. This difference may be due to the fact that the data in NCRB is deaths by suicide which has high intent to die, and so the hanging tops in that list. But in this study, poisoning has high percentage probably due to low intent.

In further assessing the type of poisoning, rat killer poison and abrus seed poison shares $26.1 \%$ each among suicide attempt by poisoning. Remaining types of poisoning used for suicide attempt by subjects in this study are, ant killer poison $14.5 \%$, kerosene $11.6 \%$, oleander seed poison in $8.7 \%$, abrus seed poison in $5.8 \%$. 
With the help of becks suicide intent scale the intent of the suicide attempters were assessed and found that most of the subjects had low intent (46.7\%), medium intent were seen in $40 \%$ and severe intent were seen in $13.3 \%$. It does not coincides with the data by Alvi T et al., in $2017^{9}$, which shows major of the suicide attempters in high intent category. And as we discussed earlier this low intent in suicide attempt is the reason that the mode of attempt data doesn't match with NCRB data.

\section{Conclusion}

Contrast to some data available, the result of this study shows that the intent in suicide attempters is mostly low. Which shows that the intent in suicide attempters is different from people who die of suicide, where the intent is high. The mode of attempt also differs with some study and shows that the poisoning is the major mode of attempt. In type of poisoning, this data clearly shows that the poisons that are available in house for domestic purposes such as ant killer or rat killer poison, kerosene occupies the major portion of the poisoning that are used by suicide attempters. This rings the warning sign to limit the accessibility of these types of poisons from households.

\section{Limitations}

This study does not include the data of the people died of suicide. Comparing it with the suicide attempters would have given a clear picture of difference in intent between the two. It could have also shown other modes of suicide such as drowning, firearms etc.

\section{References}

1. Accidents and suicide deaths in India : NCRB 2018 report https:// ncrb.gov.in /en/ accidental-deaths-suicides-in-india

2. Radhakrishnan R, Andrade C. Suicide: An Indian perspective. Indian J Psychiatry. 2012 Oct; 54(4):304-19. doi: 10.4103/00195545.104793. PMID: 23372232; PMCID: PMC 3554961.
3. Srivastava MK, Sahoo RN, Ghotekar LH, Dutta S, Danabalan M, Dutta TK, Das AK. Risk factors associated with attempted suicide: a case control study. Indian J Psychiatry. 2004 Jan; 46(1):33-8. PMID: 21206774; PMCID: PMC2912675.

4. Sudhir Kumar CT, Mohan R, Ranjith G, Chandrasekaran R. Gender differences in medically serious suicide attempts: a study from south India. Psychiatry Res. 2006 Sep 30; 144(1):79-86. doi: 10.1016/j.psychres.2005.11.012. Epub 2006 Aug 17. PMID: 16919336.

5. Kar N. Profile of risk factors associated with suicide attempts: A study from Orissa, India. Indian J Psychiatry. 2010 Jan; 52(1):48-56. doi: 10.4103/0019-5545.58895. PMID: 20174518 ; PMCID: PMC2824981.

6. Avril Maddrell (she/her/hers) (2020) Feminist Spaces: Gender and Geography in a Global Context, International Feminist Journal of Politics, 22:3, 451-452, DOI: 10.1080/14616742.2020.1763826

7. Palmer SJ. Comptes rendus / Reviews of books: Sati: Historical and Phenomenological Essays Arvind Sharma, editor Delhi: Motilal Banarsidass, 1988. xviii +129 p. Studies in Religion/Sciences Religieuses. 1990; 19(3):373-374. doi: $10.1177 / 000842989001900317$

8. Bastia BK, Kar N. A psychological autopsy study of suicidal hanging from Cuttack, India: focus on stressful life situations. Arch Suicide Res. 2009;13(1):100-4. doi: 10.1080/13811110802572221. PMID: 19123113.

9. Alvi T, Yasein S, Hussain S, Farjam A, Azhar M, Assad F. Attempted Suicide and Seriousness of Intent. J Coll Physicians Surg Pak. 2017 Jan;27(1):55-56. PMID: 28292373.

10. O'Connell H, Chin AV, Cunningham C, Lawlor BA. Recent developments: suicide in older people. BMJ. 2004 Oct 16;329(7471): 895-9. doi: 10.1136/bmj.329.7471.895. PMID: 15485967; PMCID: PMC523116. 
11. Crump C, Sundquist K, Sundquist J, Winkleby MA. Sociodemographic, psychiatric and somatic risk factors for suicide: a Swedish national cohort study. Psychol Med. 2014 Jan;44(2):279-89. doi: 10.1017/S0033291713000810. Epub 2013 Apr 23. PMID: 23611178.

12. Woo S, Lee SW, Lee K, Seo WS, Lee J, Kim HC, Won S. Characteristics of High-Intent Suicide Attempters Admitted to Emergency Departments. J Korean Med Sci. 2018 Sep 6;33(41):e259. doi: 10.3346/jkms.2018.33.e259. PMID: 30288157; PMCID: PMC6170672.

13. Vadlamani LN, Gowda M. Practical implications of Mental Healthcare Act 2017: Suicide and suicide attempt. Indian $\mathbf{J}$ Psychiatry. 2019 Apr;61(Suppl 4):S750-S755. doi:

10.4103/psychiatry.IndianJPsychiatry_116_19 . PMID: 31040468; PMCID: PMC6482674.

14. Dandona R, Bertozzi-Villa A, Kumar GA, Dandona L. Lessons from a decade of suicide surveillance in India: who, why and how? Int J Epidemiol. 2017 Jun 1;46(3):983-993. doi: 10.1093/ije/dyw113. PMID: 27255440. 